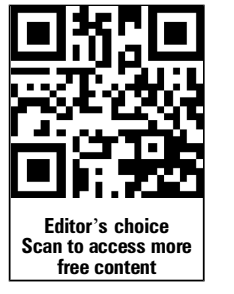

Additional material is published online only. To view please visit the journal online (http://dx.doi.org/10.1136/ heartjnl-2014-305490).

${ }^{1}$ Department of Vascular Medicine, University Medical Centre Utrecht, Utrecht, The Netherlands

${ }^{2}$ Julius Centre for Health Sciences and Primary Care, University Medical Centre Utrecht, Utrecht,

The Netherlands ${ }^{3}$ Department of Cardiology, University Medical Centre Utrecht, Utrecht, The Netherlands ${ }^{4}$ Department of Surgery, University Medical Centre Utrecht, Utrecht, The Netherlands ${ }^{5}$ Department of Neurology, University Medical Centre Utrecht, Utrecht, The Netherlands

\section{Correspondence to} Dr F L J Visseren,

Department of Vascular Medicine, University Medical Centre Utrecht, PO Box 85500, Utrecht 3508 GA

The Netherlands;

f.l.j.visseren@umcutrecht.nl

Received 5 January 2014 Revised 19 March 2014 Accepted 8 April 2014 Published Online First 9 May 2014

\section{SLinked}

- http://dx.doi.org/10.1136/ heartjnl-2014-306009

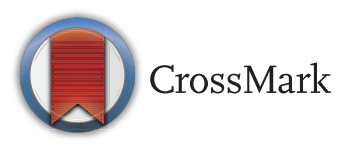

To cite: van der Leeuw J, van der Graaf $Y$,

Nathoe $\mathrm{HM}$, et al. Heart 2014; 100:1421-1429.

\title{
The separate and combined effects of adiposity and cardiometabolic dysfunction on the risk of recurrent cardiovascular events and mortality in patients with manifest vascular disease
}

\author{
J van der Leeuw, ${ }^{1}$ Y van der Graaf, ${ }^{2}$ H M Nathoe, ${ }^{3} \mathrm{G}$ J de Borst, ${ }^{4} \mathrm{~L} J$ Kappelle, $^{5}$ \\ $\mathrm{F} L \mathrm{~J}$ Visseren, ${ }^{1}$ on behalf of the SMART study group
}

\section{ABSTRACT \\ Objectives A remarkable variation exists in the} cardiometabolic consequences of obesity. We evaluated the separate and combined effects of adiposity and cardiometabolic dysfunction on the occurrence of cardiovascular events and mortality in patients with vascular disease.

Methods We prospectively followed 5231 patients with a history of clinical cardiovascular disease without diabetes from the Second Manifestations of ARTerial disease (SMART) study. Patients were classified according to body mass index and cardiometabolic function. The presence of cardiometabolic dysfunction was defined as $\geq 3$ of the modified National Cholesterol Education Program (NCEP) metabolic syndrome criteria (waist circumference replaced by elevated C-reactive protein). Cox proportional-hazards analysis was used to estimate HRs for cardiovascular events and mortality.

Results The prevalence of cardiometabolic dysfunction was $40 \%$ in normal weight, $58 \%$ in overweight and $75 \%$ in obese patients. During a median follow-up of 6.1 years, 769 patients died and 705 patients experienced a major cardiovascular event. In the absence of cardiometabolic dysfunction, overweight (HR 1.18, 95\% Cl 0.90 to 1.55) and obese patients (HR 0.93, $95 \% \mathrm{Cl} 0.53$ to 1.64 ) were not at increased risk of recurrent major cardiovascular events compared with normal weight patients without cardiometabolic dysfunction. An increased cardiovascular risk was observed in patients with cardiometabolic dysfunction and normal weight (HR 1.58,95\% Cl 1.23 to 2.04), overweight (HR 1.35, 95\% Cl 1.07 to 1.70$)$ and obesity (HR 1.50, 95\% Cl 1.12 to 2.00) compared with normal weight patients without cardiometabolic dysfunction. A similar pattern was observed for vascular and all-cause mortality.

Conclusions In patients with vascular disease, the cardiometabolic consequences of adiposity rather than adiposity per se increase the risk of recurrent cardiovascular events and mortality.

\section{INTRODUCTION}

Obesity is a growing worldwide health problem associated with excess risk of morbidity and mortality. ${ }^{1}$ Obesity relates to metabolic changes such as dyslipidemia, insulin resistance, hypertension and an inflammatory state that increase the risk of cardiovascular disease (CVD) and type 2 diabetes. ${ }^{2}$ A remarkable variation exists in the cardiometabolic consequences of obesity. Some obese patients have minor or no metabolic abnormalities, the so-called metabolically healthy obese, ${ }^{3}$ whereas normal weight individuals may suffer from numerous metabolic disturbances typically seen in obesity, the so-called metabolically obese with normal weight. ${ }^{4}$ As much as $30 \%$ of overweight and obese individuals are metabolically healthy and up to $24 \%$ of normal weight adults are metabolically abnormal. $^{3} 5$ Different criteria have been used to define such phenotypes, but all refer to a common set of traditional cardiovascular risk factors, inflammatory markers and markers of insulin resistance. ${ }^{5}$ The heterogeneity in obesity subtypes has been related to differences in body fat distribution, showing higher intra-abdominal fat content in metabolically abnormal compared with metabolically normal obese individuals. ${ }^{3}$ Accumulation of intra-abdominal fat is known to be particularly detrimental and precedes adipose tissue dysfunction, resulting in impaired insulin signalling, disordered lipid metabolism and increased secretion of proinflammatory cytokines. ${ }^{7} 8$ Furthermore, abnormal metabolic profiles have been linked to lower birth weight, later onset of obesity and decreased cardiorespiratory fitness. ${ }^{5} 9$

The clinical impact of metabolic phenotypes is underscored by prospective studies demonstrating that obesity in the absence of cardiometabolic dysfunction is not, or to a lesser extent, associated with increased cardiovascular risk in populations without prevalent diseases. ${ }^{10-12}$ In patients in the chronic phase of vascular disease, the effect of adiposity subtypes is unknown and obesity is not or even inversely related to recurrent CVD risk or mortality, referred to as the obesity paradox. ${ }^{13}$ However, risk factor clustering showed to increase the risk of new events in vascular patients. ${ }^{14} \mathrm{We}$ hypothesise that the cardiometabolic consequences of adiposity and not adiposity per se explain the variation in cardiovascular risk associated with obesity in patients with vascular disease. Therefore, the aim of the present study is to determine the separate and combined effects of adiposity and cardiometabolic dysfunction on the occurrence of cardiovascular events and mortality in vascular patients. 


\section{METHODS}

\section{Study population}

The Secondary Manifestations of ARTerial disease (SMART) study is an ongoing prospective single-centre cohort study at the University Medical Centre Utrecht, the Netherlands. Study patients were newly referred to the hospital with manifest atherosclerotic disease or for the management of cardiovascular risk factors (ie, hypertension, hyperlipidaemia or diabetes). Patients were screened for manifestations of atherosclerotic diseases and risk factors other than the qualifying diagnosis. Exclusion criteria were age under 18 years, malignancy, dependency in daily activities and insufficient fluency in the Dutch language. The Ethics Committee of the University Medical Centre Utrecht approved the study, and all participants gave their written informed consent. A detailed description of the study has been published previously. ${ }^{15}$

For the current study, we used a sample of the 9737 patients enrolled between 1996 and 2012. We selected 5431 patients with either a history or recent diagnosis of clinically manifest vascular disease without diabetes. Diabetes was defined as a referral diagnosis of diabetes, self-reported diabetes, the use of glucose-lowering agents or a baseline plasma glucose concentration of $\geq 7.0 \mathrm{mmol} / \mathrm{L}$ with initiation of glucose-lowering treatment within 1 year after inclusion. We excluded patients with diabetes as they were considered to be at the far end of cardiometabolic dysregulation and have a well-established increased risk of cardiovascular events. Patients with a body mass index (BMI) $<20 \mathrm{~kg} / \mathrm{m}^{2}(\mathrm{n}=145)$ were excluded as the risk of mortality strongly increases with lower BMI in patients with vascular disease. Lastly, patients with high-sensitive C-reactive protein (hsCRP) $>20 \mathrm{mg} / \mathrm{L}(\mathrm{n}=54)$ were excluded as these hsCRP levels more likely reflect an acute inflammatory response rather than chronic low-grade inflammation associated with adipose tissue dysfunction. The remaining 5232 patients were eligible for analyses; 3120 patients had coronary heart disease, 1531 cerebrovascular disease, 972 peripheral arterial disease and 464 abdominal aortic aneurysm. A total of 855 patients suffered from multiple sorts of vascular disease.

\section{Baseline examination}

Participants completed a questionnaire on cardiovascular history, risk factors, medication use and physical activity. BMI, weight in kilograms divided by the square of height in metres, was computed after a standardised anthropometric measurement protocol. Waist circumference was measured halfway between the lower rib and the iliac crest. Fasting venous blood samples were taken to determine serum lipids, glucose, creatinine and hsCRP levels. The techniques used for the laboratory tests have been described previously. ${ }^{15}$ Additionally, visceral and subcutaneous abdominal fat were measured in a subset ( $81 \%$ of study population) using ultrasonography, a technique validated for this purpose. ${ }^{16}$ Visceral fat was estimated by measuring the distance between the peritoneum and the lumbar spine or psoas muscles. The distance between the linea alba and the skin was used to estimate the amount of subcutaneous fat. Each distance was measured three times at three different positions providing a mean that was used in the analyses.

\section{Definitions}

Adiposity subtypes were based on the presence or absence of cardiometabolic dysfunction and BMI level. Cardiometabolic dysfunction was defined as the presence of three or more of five cardiometabolic risk factors: elevated blood pressure
( $\geq 130 \mathrm{~mm} \mathrm{Hg}$ systolic or $\geq 85 \mathrm{~mm} \mathrm{Hg}$ diastolic or use of blood pressure-lowering agents), hypertriglyceridaemia $(\geq 1.70 \mathrm{mmol} / \mathrm{L}$ or treatment for elevated triglycerides), low high-density lipoprotein (HDL)-cholesterol $(<1.03 \mathrm{mmol} / \mathrm{L}$ for men and $<1.30$ in women), a high fasting glucose $(\geq 5.6 \mathrm{mmol} / \mathrm{L}$ or use of glucose-lowering agents) or elevated hsCRP $(\geq 2 \mathrm{mg} / \mathrm{L})$. The first four criteria are similar to the National Cholesterol Education Program (NCEP) revised criteria, ${ }^{17}$ whereas waist circumference was replaced by elevated hsCRP. Normal weight was defined as a BMI of $\geq 20$ and $<25 \mathrm{~kg} / \mathrm{m}^{2}$, overweight as a BMI $\geq 25$ and $<30 \mathrm{~kg} / \mathrm{m}^{2}$ and obesity as a BMI $\geq 30 \mathrm{~kg} / \mathrm{m}^{2}$. An integrated measure of physical activity was calculated based on type and duration of physical activity. The time spent on a specific activity per week was multiplied by its metabolic equivalent intensity level (metabolic equivalent of task (MET) $)^{18}$ and summed if more than one type of activity was reported.

\section{Follow-up and clinical endpoints}

Patients were biannually asked to complete a questionnaire on hospitalisations and outpatient clinic visits. Outcomes of interest for this study were myocardial infarction, stroke, vascular death, major cardiovascular events as a composite of these three and all-cause mortality. Definitions of events are shown in online supplementary appendix 1 . When a possible event was reported, hospital discharge letters and results of relevant laboratory and radiology examinations were collected. Death and cause of death were reported by relatives of the participant, the general practitioner or the medical specialist. All events were adjudicated by three members of the SMART study Endpoint Committee, comprising physicians from different departments. Follow-up duration was defined as the period between study inclusion and first cardiovascular event or death from any cause, date of loss to follow-up or the preselected date of 1 March 2012. Of the 5232 participants, 187 (3.6\%) were lost to follow-up due to migration or discontinuation of the study.

\section{Data analyses}

Central estimators and variance measures were calculated for baseline characteristics. Normally distributed data were compared using Student's t-test, skewed data with the MannWhitney test and proportions with the $\chi^{2}$ test. Subsequently, Cox proportional hazards analysis was used to estimate HRs and $95 \%$ CIs for the occurrence of new vascular events and mortality associated with adiposity subtypes. If a patient had multiple events, the first recorded event was used. The age and gender-adjusted associations were examined in model I. To account for other extraneous risk factors for atherosclerosis, current smoking, current alcohol consumption, physical activity (low, moderate or high), use of lipid-lowering agents, use of antiplatelet agents, years since first vascular event and number of affected vascular territories (one or more) were additionally included in model II. The proportional hazards assumption was verified by correlating Schoenfeld's residuals with time and no departures from proportionality were observed. Effect modification between age and both BMI and cardiometabolic dysfunction was evaluated. We further estimated the effect of cardiometabolic dysfunction independent of BMI and aforementioned covariables. Next, the effect of an increase in level of cardiometabolic dysfunction was studied by grouping individuals according to the number of cardiometabolic risk factors $(0-1$, $2-3$ or $4-5$ ). For this purpose, overweight and obese patients were combined to avoid subgroups with too little events. Sensitivity analyses were performed to compare classification according to the modified and the original NCEP-revised 
Table 1 Baseline characteristics $(n=5232)$

\begin{tabular}{|c|c|c|c|c|c|c|c|c|c|}
\hline & \multicolumn{3}{|l|}{ Normal weight } & \multicolumn{3}{|l|}{ Overweight } & \multicolumn{3}{|l|}{ Obesity } \\
\hline & Without CMD $(n=1056)$ & With CMD $(n=717)$ & $p$ Value & Without CMD ( $n=1084)$ & With CMD ( $n=1524)$ & $p$ Value & Without CMD ( $n=212)$ & With CMD ( $n=639)$ & $\mathrm{p}$ Value \\
\hline Proportion of BMI category (\%) & 60 & 40 & & 42 & 58 & & 25 & 75 & \\
\hline Age (years)* & $59 \pm 11$ & $61 \pm 10$ & $<0.01$ & $59 \pm 11$ & $60 \pm 10$ & 0.02 & $58 \pm 11$ & $58 \pm 10$ & 0.40 \\
\hline Females (\%) & $233(31)$ & $193(27)$ & 0.12 & $226(21)$ & $308(20)$ & 0.73 & $76(36)$ & $201(32)$ & 0.27 \\
\hline BMI $\left(\mathrm{kg} / \mathrm{m}^{2}\right)^{*}$ & $23.1 \pm 1.3$ & $23.3 \pm 1.3$ & $<0.01$ & $27.0 \pm 1.3$ & $27.3 \pm 1.4$ & $<0.01$ & $32.6 \pm 2.8$ & $33.1 \pm 2.8$ & 0.03 \\
\hline \multicolumn{10}{|l|}{ Metabolic dysfunction criteria } \\
\hline Systolic blood pressure $(\mathrm{mm} \mathrm{Hg})^{*}$ & $137 \pm 22$ & $141 \pm 21$ & $<0.01$ & $138 \pm 20$ & $142 \pm 21$ & $<0.01$ & $140 \pm 19$ & $142 \pm 20$ & 0.20 \\
\hline Diastolic blood pressure $(\mathrm{mm} \mathrm{Hg})^{*}$ & $80 \pm 12$ & $81 \pm 12$ & 0.06 & $82 \pm 11$ & $82 \pm 11$ & 0.40 & $83 \pm 11$ & $84 \pm 12$ & 0.42 \\
\hline Triglycerides $(\mathrm{mmol} / \mathrm{L}) \dagger$ & $1.0(0.8-1.3)$ & $1.7(1.1-2.3)$ & $<0.01$ & $1.1(0.9-1.4)$ & $1.8(1.3-2.5)$ & $<0.01$ & $1.1(0.9-1.4)$ & $1.8(1.4-2.5)$ & $<0.01$ \\
\hline HDL-cholesterol (mmol/L)* & $1.5 \pm 0.4$ & $1.1 \pm 0.3$ & $<0.01$ & $1.4 \pm 0.3$ & $1.1 \pm 0.3$ & $<0.01$ & $1.4 \pm 0.3$ & $1.1 \pm 0.3$ & $<0.01$ \\
\hline Fasting glucose* & $5.4 \pm 0.6$ & $5.9 \pm 0.7$ & $<0.01$ & $5.5 \pm 0.6$ & $6.0 \pm 0.7$ & $<0.01$ & $5.7 \pm 0.7$ & $6.2 \pm 0.9$ & $<0.01$ \\
\hline hs-CRP (mg/L)† & $1.0(0.5-1.9)$ & $3.3(1.6-5.7)$ & $<0.01$ & $1.2(0.7-1.9)$ & $3.0(1.8-5.1)$ & $<0.01$ & $1.4(0.8-2.4)$ & $3.4(1.8-6.2)$ & $<0.01$ \\
\hline \multicolumn{10}{|l|}{ Other risk factors } \\
\hline LDL-cholesterol $(\mathrm{mmol} / \mathrm{L})^{*}$ & $2.9 \pm 1.0$ & $3.2 \pm 1.1$ & $<0.01$ & $2.8 \pm 1.0$ & $3.1 \pm 1.0$ & $<0.01$ & $2.6 \pm 0.9$ & $3.0 \pm 1.0$ & $<0.01$ \\
\hline Current smoking, n(\%) & $321(30)$ & $346(48)$ & $<0.01$ & $271(25)$ & $521(34)$ & $<0.01$ & $55(26)$ & $230(36)$ & $<0.01$ \\
\hline Current alcohol consumption, n(\%) & $614(58)$ & $301(42)$ & $<0.01$ & $704(65)$ & $721(47)$ & $<0.01$ & $127(60)$ & $314(49)$ & $<0.01$ \\
\hline Physical activity (hours ${ }^{*}$ MET $^{*}$ week $^{-1}$ ) $\dagger$ & $40(19-67)$ & $26(9-53)$ & $<0.01$ & $39(21-66)$ & $30(11-59)$ & $<0.01$ & $43(21-74)$ & $26(10-55)$ & $<0.01$ \\
\hline Years since first vascular event (years) $\dagger$ & $0(0-1)$ & $0(0-4)$ & 0.05 & $0(0-2)$ & $0(0-4)$ & $<0.01$ & $0(0-2)$ & $0(0-4)$ & 0.11 \\
\hline \multicolumn{10}{|l|}{ Adiposity measures } \\
\hline Waist circumference $(\mathrm{cm})^{*}$ & $86 \pm 9$ & $88 \pm 8$ & $<0.01$ & $95 \pm 8$ & $98 \pm 7$ & $<0.01$ & $106 \pm 10$ & $109 \pm 10$ & $<0.01$ \\
\hline Hip circumference $(\mathrm{cm})^{*}$ & $98 \pm 5$ & $99 \pm 5$ & 0.34 & $104 \pm 5$ & $105 \pm 5$ & $<0.01$ & $114 \pm 7$ & $114 \pm 8$ & 0.72 \\
\hline Subcutaneous fat $(\mathrm{cm})^{*} \ddagger$ & $2.1 \pm 1.0$ & $2.1 \pm 0.9$ & 0.10 & $2.5 \pm 1.1$ & $2.4 \pm 1.3$ & 0.23 & $3.2 \pm 1.4$ & $3.2 \pm 1.5$ & 0.72 \\
\hline Visceral fat $(\mathrm{cm})^{*} \ddagger$ & $7.2 \pm 1.8$ & $8.0 \pm 1.8$ & $<0.01$ & $8.7 \pm 2.0$ & $9.7 \pm 2.0$ & $<0.01$ & $10.2 \pm 2.6$ & $11.3 \pm 2.4$ & $<0.01$ \\
\hline \multicolumn{10}{|l|}{ Medications } \\
\hline Lipid-lowering agents, n(\%) & $654(62)$ & $401(56)$ & 0.01 & $752(69)$ & $984(65)$ & 0.01 & $173(82)$ & $434(68)$ & $<0.01$ \\
\hline Blood pressure-lowering agents, $n(\%)$ & $606(57)$ & $528(74)$ & $<0.01$ & $755(70)$ & $1179(77)$ & $<0.01$ & $163(77)$ & $529(83)$ & 0.07 \\
\hline Antiplatelet agents, n(\%) & $771(73)$ & $521(73)$ & 0.91 & $864(80)$ & $1153(76)$ & 0.02 & $179(84)$ & $475(75)$ & $<0.01$ \\
\hline
\end{tabular}

${ }^{*} \mathrm{Mean} \pm \mathrm{SD}$

Pearson's $r$ for BMl and subcutaneous fat was 0.32 , and for BMI and visceral fat 0.56

BMI, body mass index; CMD, cardiometabolic dysfunction; HDL, high-density lipoprotein; hs-CRP, high-sensitivity C-reactive protein, LDL, low-density lipoprotein; MET, metabolic equivalent of task. 
criteria inclusive of abdominal obesity (waist circumference $\geq 102 \mathrm{~cm}$ in men and $\geq 88 \mathrm{~cm}$ in women). ${ }^{17}$ Further, adiposity subgroups were constructed based on sex-specific tertiles of ultrasonically measured visceral fat instead of BMI.

To reduce bias and increase statistical rigour, missing values were completed by single regression imputation using predictive mean matching; fasting glucose $(n=30 ; 0.6 \%)$, triglycerides $(\mathrm{n}=34 ; 0.6 \%)$, HDL-cholesterol $(\mathrm{n}=36 ; 0.7 \%)$, systolic blood pressure $(n=37 ; 0.7 \%)$, diastolic blood pressure $(n=40 ; 0.8 \%)$, hsCRP ( $\mathrm{n}=99 ; 1.9 \%)$, physical activity $(\mathrm{n}=61 ; 1.2 \%)$, waist circumference $(\mathrm{n}=757,14.5 \%)$, BMI $(\mathrm{n}=10 ; 0.2 \%)$ and years since first vascular event $(\mathrm{n}=15 ; 0.3 \%)$. Statistical analyses were performed with the open source statistical package R, V.2.15.2.

\section{RESULTS}

\section{Baseline characteristics}

The study population comprised mainly Caucasian patients $(\geq 95 \%)$ with an average age of $61 \pm 10$ years. The majority of patients were male (75\%). Baseline characteristics stratified according to adiposity and cardiometabolic dysfunction are presented in table 1. Within these strata, BMI was comparable among patients with and without cardiometabolic dysfunction. The prevalence of cardiometabolic dysfunction was $40 \%$ $(\mathrm{n}=717)$ in normal weight patients, 58\% $(\mathrm{n}=1524)$ in overweight and $75 \%(n=639)$ in obese patients. During a median follow-up of 6.1 years (IQR 3.1-9.3 years) with a total follow-up of 33797 person-years, 769 participants died, of whom 399 died from a vascular cause. The composite of major cardiovascular events occurred in 705 participants, 376 experienced a myocardial infarction and 214 a stroke.

\section{Risk of adiposity subtypes on cardiovascular events and all-cause mortality}

In the absence of cardiometabolic dysfunction, overweight (HR $1.18,95 \%$ CI 0.90 to 1.55 ) and obese patients (HR $0.93,95 \%$ CI 0.53 to 1.64 ) did not have an increased risk of major cardiovascular events compared with normal weight patients without cardiometabolic dysfunction (table 2). Similarly, overweight (HR 1.03, 95\% CI 0.80 to 1.33) and obese patients (HR 0.88, $95 \% \mathrm{CI} 0.51$ to 1.53 ) without cardiometabolic dysfunction did not appear to be at increased risk of all-cause mortality (table 3). Alternatively, normal weight patients with cardiometabolic dysfunction were at increased risk of major cardiovascular events (HR 1.58, 95\% CI 1.23 to 2.04 ) and all-cause mortality (HR 1.41, 95\% CI 1.12 to 1.78 ) compared with normal weight patients without cardiometabolic dysfunction (tables 2 and 3).

Table 2 The risk of adiposity in the presence or absence of cardiometabolic dysfunction on recurrent cardiovascular events

\begin{tabular}{|c|c|c|c|}
\hline & Events/n & Age and sex adjusted HR & Multivariable adjusted $\mathrm{HR}^{*}$ \\
\hline \multicolumn{4}{|c|}{ Major cardiovascular events $\dagger$} \\
\hline \multicolumn{4}{|c|}{ Normal weight } \\
\hline Without CMD & $102 / 1056$ & 1 & 1 \\
\hline With CMD & $150 / 717$ & $1.93(1.50$ to 2.48$)$ & 1.58 (1.23 to 2.04$)$ \\
\hline \multicolumn{4}{|l|}{ Overweight } \\
\hline Without CMD & $109 / 1084$ & 1.13 (0.86 to 1.48$)$ & $1.18(0.90$ to 1.55$)$ \\
\hline With CMD & $243 / 1524$ & $1.53(1.22$ to 1.93$)$ & 1.35 (1.07 to 1.70$)$ \\
\hline \multicolumn{4}{|l|}{ Obese } \\
\hline Without CMD & $14 / 212$ & 0.85 (0.49 to 1.49$)$ & 0.93 (0.53 to 1.64$)$ \\
\hline With CMD & $87 / 639$ & 1.66 (1.24 to 2.21$)$ & 1.50 (1.12 to 2.00$)$ \\
\hline \multicolumn{4}{|c|}{ Myocardial infarction } \\
\hline \multicolumn{4}{|l|}{ Normal weight } \\
\hline Without CMD & $47 / 1056$ & 1 & 1 \\
\hline With CMD & $72 / 717$ & 2.03 (1.41 to 2.93$)$ & 1.68 (1.16 to 2.44$)$ \\
\hline \multicolumn{4}{|l|}{ Overweight } \\
\hline Without CMD & $61 / 1084$ & 1.31 (0.89 to 1.92$)$ & 1.33 (0.91 to 1.95$)$ \\
\hline With CMD & $141 / 1524$ & 1.87 (1.35 to 2.61$)$ & 1.62 (1.16 to 2.26$)$ \\
\hline \multicolumn{4}{|l|}{ Obese } \\
\hline Without CMD & $5 / 212$ & 0.65 (0.26 to 1.62$)$ & $0.69(0.27$ to 1.73$)$ \\
\hline With CMD & $50 / 639$ & 1.94 (1.30 to 2.89$)$ & 1.69 (1.13 to 2.53$)$ \\
\hline \multicolumn{4}{|l|}{ Stroke } \\
\hline \multicolumn{4}{|l|}{ Normal weight } \\
\hline Without CMD & $33 / 1056$ & 1 & 1 \\
\hline With CMD & $47 / 717$ & 1.90 (1.21 to 2.96$)$ & 1.58 (1.01 to 2.48$)$ \\
\hline \multicolumn{4}{|l|}{ Overweight } \\
\hline Without CMD & $37 / 1084$ & $1.18(0.74$ to 1.89$)$ & $1.25(0.78$ to 2.01$)$ \\
\hline With CMD & $65 / 1524$ & 1.28 (0.84 to 1.94$)$ & 1.17 (0.77 to 1.78$)$ \\
\hline \multicolumn{4}{|l|}{ Obese } \\
\hline Without CMD & $7 / 212$ & 1.28 (0.56 to 2.89$)$ & $1.36(0.60$ to 3.08$)$ \\
\hline With CMD & $25 / 639$ & $1.42(0.84$ to 2.40$)$ & $1.33(0.79$ to 2.25$)$ \\
\hline
\end{tabular}


Table 3 The risk of adiposity in the presence or absence of cardiometabolic dysfunction on vascular and all-cause mortality

\begin{tabular}{|c|c|c|c|}
\hline & Events/n & Age and sex adjusted HR & Multivariable adjusted $\mathrm{HR}^{*}$ \\
\hline \multicolumn{4}{|c|}{ All-cause mortality } \\
\hline \multicolumn{4}{|l|}{ Normal weight } \\
\hline Without CMD & $128 / 1056$ & 1 & 1 \\
\hline With CMD & $176 / 717$ & 1.73 (1.38 to 2.17$)$ & 1.41 (1.12 to 1.78$)$ \\
\hline \multicolumn{4}{|l|}{ Overweight } \\
\hline Without CMD & $114 / 1084$ & $1.00(0.78$ to 1.29$)$ & $1.03(0.80$ to 1.33$)$ \\
\hline With CMD & $252 / 1524$ & 1.27 (1.03 to 1.57$)$ & $1.13(0.91$ to 1.40$)$ \\
\hline \multicolumn{4}{|l|}{ Obese } \\
\hline Without CMD & $14 / 212$ & 0.81 (0.47 to 1.41 ) & $0.88(0.51$ to 1.53$)$ \\
\hline With CMD & $85 / 639$ & 1.51 (1.15 to 2.00$)$ & 1.37 (1.04 to 1.82$)$ \\
\hline \multicolumn{4}{|l|}{ Vascular mortality } \\
\hline \multicolumn{4}{|l|}{ Normal weight } \\
\hline Without CMD & $56 / 1056$ & 1 & 1 \\
\hline With CMD & $100 / 717$ & 2.26 (1.63 to 3.14$)$ & 1.74 (1.25 to 2.42$)$ \\
\hline \multicolumn{4}{|l|}{ Overweight } \\
\hline Without CMD & $57 / 1084$ & 1.14 (0.79 to 1.65$)$ & $1.18(0.82$ to 1.71$)$ \\
\hline With CMD & $131 / 1524$ & 1.52 (1.11 to 2.09$)$ & 1.29 (0.94 to 1.77$)$ \\
\hline \multicolumn{4}{|l|}{ Obese } \\
\hline Without CMD & $8 / 212$ & 1.05 (0.50 to 2.21$)$ & $1.20(0.57$ to 2.53$)$ \\
\hline With CMD & $47 / 639$ & 1.94 (1.31 to 2.87$)$ & 1.70 (1.15 to 2.52$)$ \\
\hline
\end{tabular}

Irrespective of adiposity, the presence of cardiometabolic dysfunction increased the risk of major cardiovascular events (HR $1.35,95 \%$ CI 1.15 to 1.60$)$ and mortality (HR 1.26, 95\% CI
1.08 to 1.48). Similar trends were observed for myocardial infarction, stroke and vascular mortality (tables 2 and 3). Age did not modify these relations $(\mathrm{p}>0.11)$.

Table 4 The risk of adiposity in the presence or absence of the traditional metabolic syndrome on recurrent vascular events and mortality

\begin{tabular}{|c|c|c|c|}
\hline & Events/n & Age and sex adjusted HR & Multivariable adjusted $\mathrm{HR}^{*}$ \\
\hline \multicolumn{4}{|c|}{ Major cardiovascular events $\dagger$} \\
\hline \multicolumn{4}{|l|}{ Normal weight } \\
\hline Without MetS & $155 / 1299$ & 1 & 1 \\
\hline With MetS & $97 / 474$ & 1.59 (1.23 to 2.05$)$ & $1.43(1.11$ to 1.85$)$ \\
\hline \multicolumn{4}{|l|}{ Overweight } \\
\hline Without MetS & $146 / 1207$ & 1.08 (0.86 to 1.35$)$ & 1.13 (0.90 to 1.42$)$ \\
\hline With MetS & 206/1401 & $1.20(0.98$ to 1.48$)$ & 1.15 (0.93 to 1.42$)$ \\
\hline \multicolumn{4}{|l|}{ Obese } \\
\hline Without MetS & 9/129 & 0.74 (0.38 to 1.44$)$ & 0.85 (0.43 to 1.67$)$ \\
\hline With MetS & $92 / 722$ & 1.31 (1.01 to 1.69 ) & 1.28 (0.99 to 1.67$)$ \\
\hline \multicolumn{4}{|c|}{ All-cause mortality } \\
\hline \multicolumn{4}{|l|}{ Normal weight } \\
\hline Without MetS & $188 / 1299$ & 1 & 1 \\
\hline With MetS & $116 / 474$ & 1.48 (1.17 to 1.87$)$ & 1.37 (1.08 to 1.73$)$ \\
\hline \multicolumn{4}{|l|}{ Overweight } \\
\hline Without MetS & $157 / 1207$ & $1.00(0.81$ to 1.23$)$ & $1.05(0.85$ to 1.30$)$ \\
\hline With MetS & $209 / 1401$ & 1.02 (0.84 to 1.24$)$ & $1.00(0.82$ to 1.21$)$ \\
\hline \multicolumn{4}{|l|}{ Obese } \\
\hline Without MetS & $8 / 129$ & 0.63 (0.31 to 1.28$)$ & $0.74(0.36$ to 1.50$)$ \\
\hline With MetS & $91 / 722$ & 1.26 (0.98 to 1.63$)$ & 1.25 (0.97 to 1.62$)$ \\
\hline
\end{tabular}


Classification of adiposity subtypes according to the traditional metabolic syndrome definition showed similar but attenuated effects on risk of major cardiovascular events and mortality (table 4). Further, categorisation of adiposity subtypes based on visceral fat showed a trend towards higher risk of major cardiovascular events across tertiles, both in the presence and absence of cardiometabolic dysfunction. Moreover, patients in the intermediate and highest tertile of visceral fat without cardiometabolic dysfunction were at significantly increased risk of all-cause mortality compared with patients in the lowest tertile (table 5).

\section{Risk of adiposity and number of cardiometabolic risk factors on cardiovascular events and all-cause mortality}

Overweight or obese patients with 0 or 1 cardiometabolic risk factor did not have an increased risk of major cardiovascular events (HR 1.23, 95\% CI 0.75 to 2.03 ) or all-cause mortality (HR 1.00, 95\% CI 0.61 to 1.64) compared with normal weight patients with 0 or 1 risk factor (figure 1). In normal weight patients, the presence of two or three risk factors was associated with a trend towards higher risk and the presence of four or five risk factors conferred an increased risk of major cardiovascular events (HR 2.25, 95\% CI 1.47 to 3.45) and all-cause mortality (HR 1.86, 95\% CI 1.26 to 2.74). Similar trends were seen for myocardial infarction, stroke and vascular mortality.

\section{DISCUSSION}

In patients with clinical vascular disease, cardiometabolic dysfunction was associated with increased risk of cardiovascular events and mortality irrespective of the presence or severity of adiposity. Overweight and obesity without cardiometabolic dysfunction did not appear to confer an increased risk of recurrent cardiovascular events and all-cause mortality.

In this study, we used a definition of cardiometabolic dysfunction based on the revised NCEP criteria for the metabolic syndrome. ${ }^{17}$ Waist circumference was omitted as it is highly correlated with BMI, which would automatically increase the number of metabolic syndrome criteria in overweight and obese individuals. ${ }^{1}$ Instead, we incorporated hsCRP in the cardiometabolic dysfunction definition as it reflects the functional and systemic consequences of excess visceral fat, typified by an increased release of free fatty acids and the secretion of proinflammatory cytokines. ${ }^{78}$ This metabolic situation is referred to as adipose tissue dysfunction and is considered to be the 'common soil' underlying the clustering of cardiometabolic risk factors. ${ }^{19}$ Further, elevated plasma concentrations of hsCRP have been directly related to an increased cardiovascular risk. ${ }^{20}$

The distinct pathophysiological role of intra-abdominal fat was underscored by a higher waist circumference and visceral fat thickness in patients with cardiometabolic dysfunction. This is not surprising given the direct association between, among others, visceral fat and hsCRP in this population. ${ }^{21}$ Log hsCRP levels increased with $0.10 \mathrm{mg} / \mathrm{L}$ in men and $0.11 \mathrm{mg} / \mathrm{L}$ in women per SD increase in visceral fat. ${ }^{21}$ Further, defining adiposity subgroups by visceral fat showed an increased risk of allcause mortality across higher tertiles, even in the absence of cardiometabolic dysfunction. These observations support the notion that both BMI and waist circumference are imperfect reflections of the detrimental visceral adipose tissue compartment. $^{22}$

This is the first study to evaluate the separate and combined effects of adiposity and cardiometabolic dysfunction in the patient with manifest vascular disease. The current findings shed

Table 5 The risk of visceral adiposity measured by ultrasound in the presence or absence of cardiometabolic dysfunction on recurrent vascular events and mortality

\begin{tabular}{|c|c|c|c|}
\hline & Events/n & Age and sex adjusted HR & Multivariable adjusted $\mathrm{HR}^{*}$ \\
\hline \multicolumn{4}{|c|}{ Major cardiovascular events $\dagger$} \\
\hline \multicolumn{4}{|c|}{ Lowest tertile } \\
\hline Without CMD & $54 / 937$ & 1 & 1 \\
\hline With CMD & $49 / 463$ & 1.70 (1.16 to 2.51$)$ & 1.44 (0.97 to 2.12$)$ \\
\hline \multicolumn{4}{|l|}{ Intermediate tertile } \\
\hline Without CMD & $43 / 640$ & $1.10(0.74$ to 1.64$)$ & $1.10(0.73$ to 1.64$)$ \\
\hline With CMD & $84 / 732$ & 1.91 (1.36 to 2.69$)$ & 1.60 (1.13 to 2.26$)$ \\
\hline \multicolumn{4}{|l|}{ Highest tertile } \\
\hline Without CMD & $33 / 425$ & $1.24(0.80$ to 1.91$)$ & $1.23(0.80$ to 1.91$)$ \\
\hline With CMD & $138 / 1022$ & 2.07 (1.51 to 2.84$)$ & $1.70(1.24$ to 2.35$)$ \\
\hline \multicolumn{4}{|l|}{ All-cause mortality } \\
\hline \multicolumn{4}{|l|}{ Lowest tertile } \\
\hline Without CMD & $43 / 937$ & 1 & 1 \\
\hline With CMD & $52 / 463$ & 2.24 (1.49 to 3.35$)$ & 1.75 (1.16 to 2.63$)$ \\
\hline \multicolumn{4}{|l|}{ Intermediate tertile } \\
\hline Without CMD & $51 / 640$ & 1.56 (1.04 to 2.34$)$ & 1.53 (1.02 to 2.29$)$ \\
\hline With CMD & $72 / 732$ & $2.00(1.37$ to 2.91$)$ & 1.62 (1.11 to 2.38$)$ \\
\hline \multicolumn{4}{|l|}{ Highest tertile } \\
\hline Without CMD & $39 / 425$ & 1.72 (1.11 to 2.65$)$ & 1.66 (1.08 to 2.57$)$ \\
\hline With CMD & $133 / 1022$ & 2.21 (1.57 to 3.12$)$ & 1.73 (1.22 to 2.46$)$ \\
\hline
\end{tabular}


A Major cardiovascular events

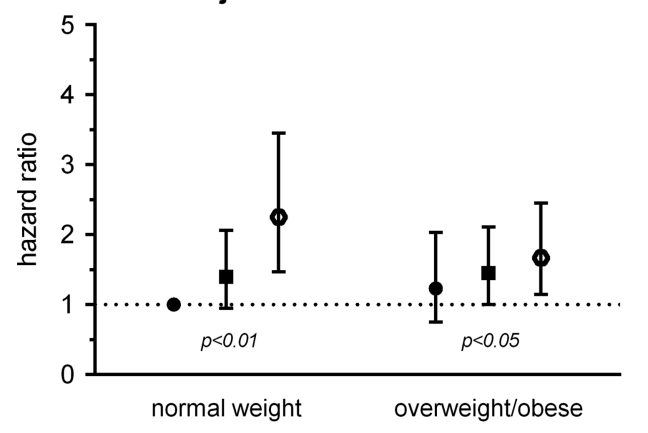

B

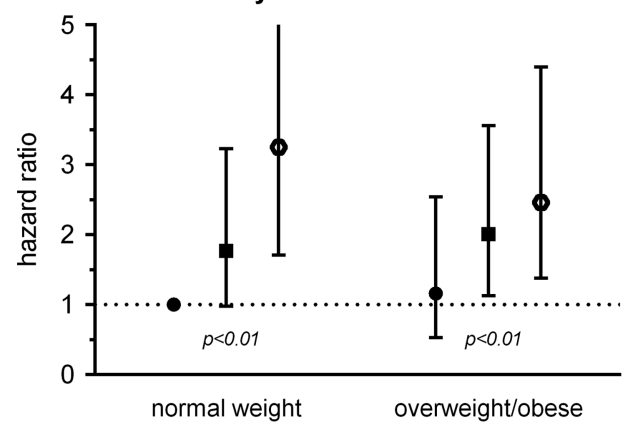

$\mathrm{D}$

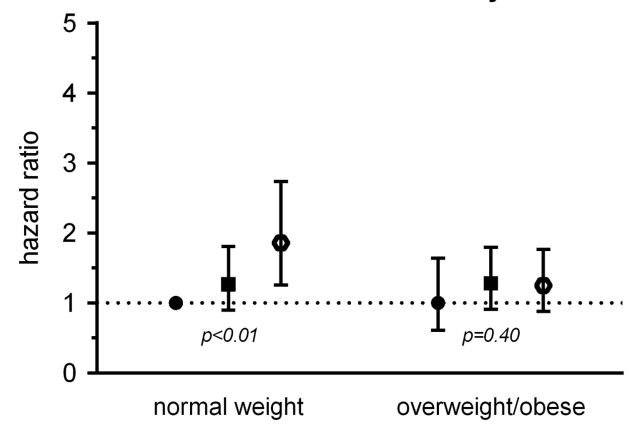

- 0-1 criteria

- 2-3 criteria

- 4-5 criteria

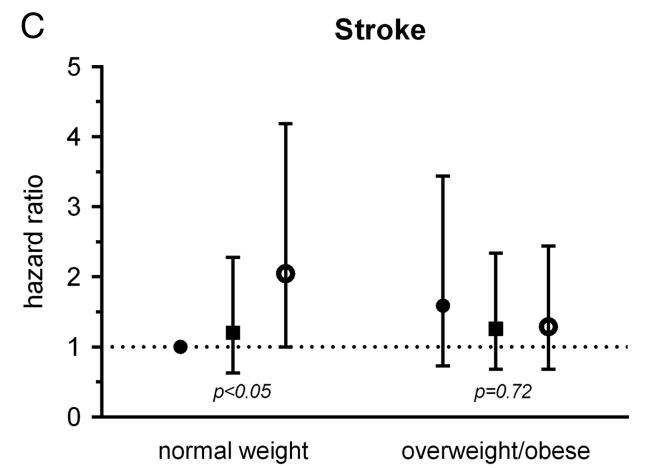

$\mathrm{E}$

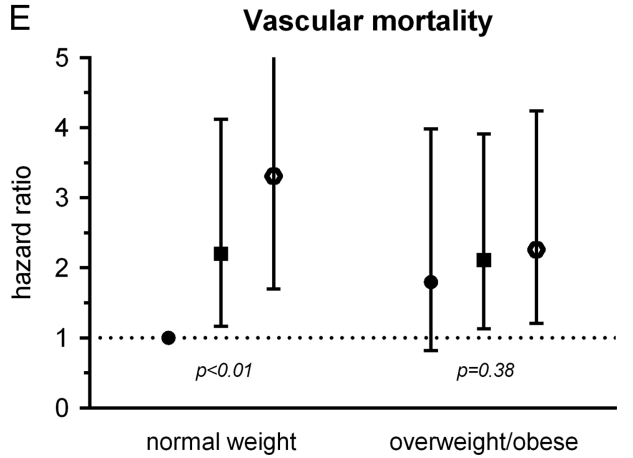

Figure 1 The risk of adiposity and level of cardiometabolic dysfunction on recurrent cardiovascular events and mortality. HRs adjusted for age, sex, current smoking, alcohol consumption, physical activity, use of lipid lowering agents, use of antiplatelet agents, years since first vascular event and number of affected vascular territories. Categorised by normal weight $\left(20-25 \mathrm{~kg} / \mathrm{m}^{2}\right)$ and overweight or obesity $\left(>25 \mathrm{~kg} / \mathrm{m}^{2}\right) ; \mathrm{p}=\mathrm{p}$ for trend.

a different light on the obesity paradox, which suggests a survival benefit for overweight and obese compared with normal weight patients with vascular disease. ${ }^{13}$ We demonstrated that a subset of overweight and obese patients, that is, those with cardiometabolic dysfunction, was at higher risk compared with normal weight individuals without cardiometabolic dysfunction. By contrast, normal weight patients with cardiometabolic dysfunction were also at high risk of cardiovascular events and mortality, thereby negating a potential risk difference with overweight and obese patients. The inverse relation between adiposity and cardiovascular risk in vascular patients may thus partly result from the differences in the cardiometabolic burden of adiposity.

Our findings are in accordance with previous longitudinal studies in populations without prevalent diseases showing that overweight and obesity in the absence of metabolic dysfunction are not associated with increased cardiovascular risk. ${ }^{10-12}$ Opposite findings were observed by others. A recent report of the Copenhagen General Population study did not find a low-risk adiposity subtype, but did not take the inflammatory component of obesity and atherosclerosis into account. ${ }^{23}$ Other studies with opposite findings did not account for the effect of physical activity. ${ }^{24} 25$ This is relevant as physical activity may be a proxy of cardiorespiratory fitness, which is associated with better prognosis. ${ }^{11}$ Two recent meta-analyses of overlapping studies evaluated the effects of adiposity subtypes and showed that metabolically healthy overweight patients were not at increased risk over a short follow-up period ( $\leq 10$ or 15 years), whereas risk was increased in studies with a long a follow-up period ( $>10$ or 15 years). ${ }^{26} 27$ The long-term results were largely driven by the study of Flint et $a l^{28}$, which defined a normal metabolic phenotype as the absence of hypercholesterolaemia, hypertension or diabetes in comparison to the much stricter definition used in present study. In another long-term study by Arnlöv et $a l^{24}$, waist circumference could not be used to define the metabolic syndrome and was replaced by BMI, which is an inaccurate measure of the abdominal adipose tissue compartment. Although we cannot rule out an increased cardiovascular risk for overweight and obese vascular patients without cardiometabolic dysfunction in the long run, it is likely that the 
existence of a low-risk phenotype depends on the criteria used to define such a subtype.

The heterogeneity in metabolic consequences of obesity may affect therapeutic decision making. For example, it was demonstrated that the benefit of weight loss on cardiovascular risk reduction is smaller for obese individuals without cardiometabolic dysfunction. ${ }^{29}{ }^{30}$ Alternatively, the steepest increase in risk associated with a greater number of cardiometabolic risk factors was observed in normal weight patients. These patients developed cardiometabolic dysfunction in the absence of excess adiposity and indicate that quantity is not the sole indicator of adipose tissue function. These patients should receive aggressive therapy to improve their level of modifiable risk factors.

Strengths and potential limitations of our study merit consideration. The strengths are the large cohort of patients with clinical vascular disease with substantial follow-up duration, high number of events and little loss to follow-up. As a limitation we consider the relatively small number of events in some subgroups that might attenuate the precision of the results and for these categories conclusion should be drawn with caution. Next, we could not account for the potentially confounding effects of dietary factors, socioeconomic status or family history of CVD. Further, results do not pertain to vascular patients with lownormal weight (ie, BMI $18.5-20 \mathrm{~kg} / \mathrm{m}^{2}$ ). These few patients $(\mathrm{n}=106)$ were not included in the reference group of normal weight patients as they were at increased vascular and mortality risk, negating potential risk differences with overweight and obese patients. Further, categorisation and analyses relied on baseline measurements while patients without cardiometabolic dysfunction could have acquired cardiometabolic abnormalities during follow-up, thereby attenuating the differences. Further,

\section{Key messages}

\section{What is already known on this subject?}

A remarkable variation exists in the cardiometabolic consequences of obesity.

Overweight and obesity are not or even inversely related to risk of recurrent cardiovascular events in patients with overt vascular disease.

Cardiometabolic risk factors increase the risk of recurrent events in patients with overt vascular disease.

\section{What this study adds?}

This study shows that variations in cardiometabolic consequences of adiposity might help to understand the paradoxical relation between adiposity and cardiovascular risk in patients with manifest vascular disease.

Overweight or obese vascular patients without cardiometabolic dysfunction were not at increased risk of recurrent cardiovascular events.

By contrast, vascular patients with cardiometabolic dysfunction were at increased risk of recurrent events irrespective of adiposity. Moreover, risk was highest in normal weight patients with cardiometabolic abnormalities.

\section{How might this impact on clinical practice?}

Risk of recurrent events is better explained by cardiometabolic dysfunction compared to adiposity per se. Physicians can better identify vascular patients at high risk of recurrent events by assessing cardiometabolic status compared with assessment of the patient's body mass index. the threshold of cardiometabolic dysfunction could be reached by various combinations of cardiometabolic criteria, translating to heterogeneity in the risk of patients with cardiometabolic dysfunction. Lastly, the risk associated with an increasing number of risk factors is likely to be a continuum rather than a single cut-off point beyond which risk suddenly rises. This continuous relation was supported by the classification of adiposity subtypes based the number of cardiometabolic risk factors.

In conclusion, cardiometabolic dysfunction was associated with increased risk of cardiovascular events and mortality irrespective of adiposity level in patients with vascular disease. Overweight and obesity without cardiometabolic dysfunction did not appear to confer an increased risk of cardiovascular events or mortality. Hence, the cardiometabolic consequences of adiposity rather than adiposity per se increase the risk of cardiovascular events and mortality in patients with vascular disease.

Acknowledgements We gratefully acknowledge the contribution of the SMART research nurses.

Collaborators R van Petersen (data-manager); A G Pij (vascular manager); and the participants of the SMART Study Group: A Algra MD, PhD; $Y$ van der Graaf, MD, PhD; D E Grobbee, MD, PhD; G E H M Rutten, MD, PhD, Julius Center for Health Sciences and Primary care; F L J Visseren, MD, PhD, Department of Internal Medicine; F L Moll, MD, PhD, Department of Vascular Surgery; L J Kappelle, MD, PhD, Department of Neurology; W P T M Mali, MD, PhD, Department of Radiology; $\mathrm{P}$ A Doevendans, MD, PhD, Department of Cardiology.

Contributors JvdL designed and carried out the data analyses, interpreted the results and drafted the manuscript. YvdG and FLJV contributed to the researched data, designed the data analyses, interpreted the results and revised the manuscript for important intellectual content. HN, GJB and LJK contributed to the research data, interpreted the results and revised the manuscript for important intellectual content. YvdG and FLJV are the guarantors of this work and, as such, had full access to all the data in the study and take responsibility for the integrity of the data and the accuracy of the data analysis.

Funding The SMART study was financially supported by a grant of the University Medical Centre Utrecht.

Competing interests None.

Patient consent Obtained.

Ethics approval The Ethics Committee of the University Medical Centre Utrecht.

Provenance and peer review Not commissioned; externally peer reviewed.

\section{REFERENCES}

1 Pischon T, Boeing H, Hoffmann $\mathrm{K}$, et al. General and abdominal adiposity and risk of death in Europe. N Engl J Med 2008;359:2105-20.

2 Van Gaal LF, Mertens IL, De Block CE. Mechanisms linking obesity with cardiovascular disease. Nature 2006:444:875-80.

3 Wildman RP, Muntner P, Reynolds K, et al. The obese without cardiometabolic risk factor clustering and the normal weight with cardiometabolic risk factor clustering: prevalence and correlates of 2 phenotypes among the US population (NHANES 1999-2004). Arch Intern Med 2008;168:1617-24.

4 Ruderman N, Chisholm D, Pi-Sunyer X, et al. The metabolically obese, normal-weight individual revisited. Diabetes 1998;47:699-713.

5 Primeau V, Coderre L, Karelis $A D$, et al. Characterizing the profile of obese patients who are metabolically healthy. Int J Obes 2011:35:971-81.

6 Brochu M, Tchernof A, Dionne IJ, et al. What are the physical characteristics associated with a normal metabolic profile despite a high level of obesity in postmenopausal women? J Clin Endocrinol Metab 2001;86:1020-5.

7 Andersson CX, Gustafson B, Hammarstedt A, et al. Inflamed adipose tissue, insulin resistance and vascular injury. Diabetes Metab Res Rev 2008;24:595-603.

8 Gustafson B. Adipose tissue, inflammation and atherosclerosis. J Atheroscler Thromb 2010;17:332-41.

9 Karelis AD, St-Pierre DH, Conus F, et al. Metabolic and body composition factors in subgroups of obesity: what do we know? J Clin Endocrinol Metab 2004;89:2569-75.

10 Meigs JB, Wilson PWF, Fox CS, et al. Body mass index, metabolic syndrome, and risk of type 2 diabetes or cardiovascular disease. J Clin Endocrinol Metab 2006;91:2906-12

11 Ortega FB, Lee DC, Katzmarzyk PT, et al. The intriguing metabolically healthy but obese phenotype: cardiovascular prognosis and role of fitness. Eur Heart $J$ 2013;34:389-97. 
12 Hamer M, Stamatakis E. Metabolically healthy obesity and risk of all-cause and cardiovascular disease mortality. J Clin Endocrinol Metab 2012;97: 2482-8.

13 Romero-Corral A, Montori VM, Somers VK, et al. Association of bodyweight with total mortality and with cardiovascular events in coronary artery disease: a systematic review of cohort studies. Lancet 2006;368:666-78.

14 Wassink AMJ, van der Graaf $Y$, Olijhoek JK, et al. Metabolic syndrome and the risk of new vascular events and all-cause mortality in patients with coronary artery disease, cerebrovascular disease, peripheral arterial disease or abdominal aortic aneurysm. Eur Heart J 2008;29:213-23.

15 Simons PC, Algra A, van de Laak MF, et al. Second manifestations of ARTerial disease (SMART) study: rationale and design. Eur J Epidemiol 1999;15:773-81.

16 Stolk RP, Wink 0, Zelissen PM, et al. Validity and reproducibility of ultrasonography for the measurement of intra-abdominal adipose tissue. Int J Obes Relat Metab Disord 2001;25:1346-51.

17 Grundy SM, Cleeman JI, Daniels SR, et al. Diagnosis and management of the metabolic syndrome: an American Heart Association/National Heart, Lung, and Blood Institute Scientific Statement. Circulation 2005;112:2735-52.

18 Ainsworth BE, Haskell WL, Whitt MC, et al. Compendium of physical activities: an update of activity codes and MET intensities. Med Sci Sports Exerc 2000;32: S498-504.

19 Hajer GR, van Haeften TW, Visseren FLJ. Adipose tissue dysfunction in obesity, diabetes, and vascular diseases. Eur Heart J 2008;29:2959-71.

20 Danesh J, Whincup $\mathrm{P}$, Walker $\mathrm{M}$, et al. Low grade inflammation and coronary heart disease: prospective study and updated meta-analyses. BMJ 2000;321:199-204.
21 Faber DR, van der Graaf $Y$, Westerink J, et al. Increased visceral adipose tissue mass is associated with increased C-reactive protein in patients with manifest vascular diseases. Atherosclerosis 2010;212:274-80.

22 Despres J-P. Body fat distribution and risk of cardiovascular disease: an update. Circulation 2012;126:1301-13.

23 Thomsen M, Nordestgaard BG. Myocardial infarction and ischemic heart disease in overweight and obesity with and without metabolic syndrome. JAMA Intern Med 2014;174:15-22

24 Arnlöv J, Ingelsson E, Sundström J, et al. Impact of body mass index and the metabolic syndrome on the risk of cardiovascular disease and death in middle-aged men. Circulation 2010;121:230-6

25 Kuk JL, Ardern $\mathrm{Cl}$. Are metabolically normal but obese individuals at lower risk for all-cause mortality? Diabetes Care 2009;32:2297-9.

26 Fan J, Song $Y$, Chen $Y$, et al. Combined effect of obesity and cardio-metabolic abnormality on the risk of cardiovascular disease: a meta-analysis of prospective cohort studies. Int J Cardiol 2013:168:4761-8.

27 Kramer CK, Zinman B, Retnakaran R. Are metabolically healthy overweight and obesity benign conditions? A systematic review and meta-analysis. Ann Intern Med 2013:159:758-69.

28 Flint AJ, Hu FB, Glynn RJ, et al. Excess weight and the risk of incident coronary heart disease among men and women. Obesity (Silver Spring) 2010;18:377-83.

29 Karelis $A D$, Messier V, Brochu $M$, et al. Metabolically healthy but obese women: effect of an energy-restricted diet. Diabetologia 2008:51:1752-4.

30 Shin M-J, Hyun YJ, Kim OY, et al. Weight loss effect on inflammation and LDL oxidation in metabolically healthy but obese $(\mathrm{MHO})$ individuals: low inflammation and LDL oxidation in MHO women. Int J Obes 2006;30:1529-34. 